Vol. 2 Issue 2, Feb.2012, pp.280-283

\title{
Cloud Computing Featuring Ontology
}

\author{
Arun Arunachalam,Shreeharsha Chandrashekar \\ Computer Engineering Department, VJTI, Matunga, Mumbai, India
}

\begin{abstract}
Mobility is about moving around. Different kinds of entities such as persons, computers, information or programs may move around. The mobile entities may communicate and access services ubiquitously or, in the contrary, services may be adapted to various characteristics of the mobility space such as the physical location. The integration of communication and computation technologies, the availability of pocket mobile computers, and the widespread penetration of mobile radio access networks will enable a range of new mobile services to be offered to the users.With the recent advances in mobile computer technology and the penetration of wireless networks, the nature of the services proposed to the users is moving towards mobile and cloud computing supported services. Our aim is to propose methods and technologies for the development of such services and the infrastructure that support them.Cloud computing, is a technology which has gained an increasing amount of attention in recent years. Mobile handheld devices, which have been highly adopted by large user groups, especially in the form of mobile phones, constitute an interesting platform for cloud computing. Cloud computing is a very well-known term these days. Cloud computing featuring Ontology provides users of mobile devices personalized services tailored to their current location. Ontology is a formal specification of a shared conceptualisation. Using the Ontology search interface, the user searches a registry of Ontologies based upon domain, keywords, category or another searchable metadata field. In general, cloud computing customers do not own the physical infrastructure, instead avoiding capital expenditure by renting usage from a third-party provider. They consume resources as a service and pay only for resources that they use. In this case user is only charged as per the messaging scheme he is using. User can access this service anywhere anytime. Since this service is deployed on cloud, it is going to offer complete location transparency. There won't be any need to maintain physical servers thus helping in cost reduction. Also the issue about storage is taken care of by the service provider itself.
\end{abstract}

Key words - Cloud Computing,Ontology, Caas(Communication as a service).

\section{Introduction}

During the last ten years, mobile handheld devices have become a part of our everyday lives. When development in information and technology enables more complicated functions and services to be integrated, the role of the mobile device as a generic tool in everyday life grows. Mobile phones are no longer used only for voice communication, but they typically employ various kinds of multimedia functionalities from camera to different types of messaging services and information sharing channels from Internet access to the mobile TV.Mobile cloud computing is a term that refers to an infrastructure where both the data storage and the data processing happen outside of the mobile device from which an application is launched. To the typical consumer, a cloud-based mobile application looks and feels just like any app purchased or downloaded from a mobile application store like iTunes. However, the app is driven from the "cloud," not from the handheld device itself. There are already a few well-known mobile cloud apps out there including Google's Gmail and Google Voice for iPhone. When launched via iPhone homescreen shortcuts, these apps perform just like any other app on the iPhone, but all of their processing power comes from the cloud. The term "cloud computing" is being bandied about a lot these days, mainly in the context of the "future of the web". But cloud computing's potential doesn't begin and end with the personal computer's transformation into a thin client - the mobile platform is going to be heavily impacted by this technology as well.You may be wondering: what does the term "mobile cloud computing" really mean? Basically, it refers to an infrastructure where both the data storage and the data processing happen outside of the mobile device. Today, there are already some good examples of mobile cloud computing applications including mobile Gmail, Google Maps, and some navigation apps. However, the majority of applications today still do most of the data storage and processing on the mobile devices themselves and not in the cloud. In a few years, that could change.

\section{A) Why Mobile Cloud Computing?}

With a Western-centric view of the world, it can sometimes be hard to remember that not everyone owns a smartphone. There are still a large number of markets worldwide where the dominant phone is a feature phone. While it's true that smartphones will grow in percentage and feature phones will become more sophisticated in time, these lowerend phones are not going away anytime soon. And it's their very existence which will help drive the mobile cloud computing trend.

\section{Existing System:}

Lets take an example of Just Dial services. To get the contact detail of any product or hotel or any other centre, first we have to call up at the Call center of the Just Dial. Then we have to speak to the operator and ask him for the required detail. The operator takes down our mobile number and sends a message back with the detail we had requested for.Similarly, taking an example of 


\section{Arun Arunachalam,Shreeharsha Chandrashekar / IOSR Journal of Engineering (IOSRJEN) \\ www.iosrjen.org \\ ISSN : 2250-3021}

Vol. 2 Issue 2, Feb.2012, pp.280-283

another system that is provided by the different mobile company operators. They have a dedicated number for such services. Like the 56789 charges us upto Rs. 3/Rs. 5 per SMS. This is usually not affordable to the common people.

\section{A) Problems in existing system:}

The drawback in the Just Dial service is that the operator might misinterpret for the information we have requested. Also, we have to answer a lot of other question like giving our email id, our business details, etc. So there is a chance of Human Error.Now, the disadvantage in the other system that is provided by the mobile operators is that we are charged alot money for it which is not affordable for a common man.

\section{PROPOSED WORK}

The proposed system consists of an SMS gateway and the mobile users. In the proposed system when the users sends an SMS requesting the contact details of a any organization/ institution or any place, the application searches through the stored data and replies to that user with the required information along with additional data about the organization in the vicinity of the requested place and of the same category.

\section{Cloud System Overview}

specification of this conceptualization - the conceptualization is specified by using particular modeling language and particular terms. Formal specification is required in order to be able to process ontologies and operate on ontologies automatically.

1. Ontology describes a domain, while a knowledge base (based on Ontology) describes particular state of affairs. Each knowledge based system or agent has its own knowledge base, and only what can be expressed using ontology can be stored and used in the knowledge base. When an agent wants to communicate to another agent, he uses the constructs from some ontology. In order to understand in communication, ontologies must be shared between agents.
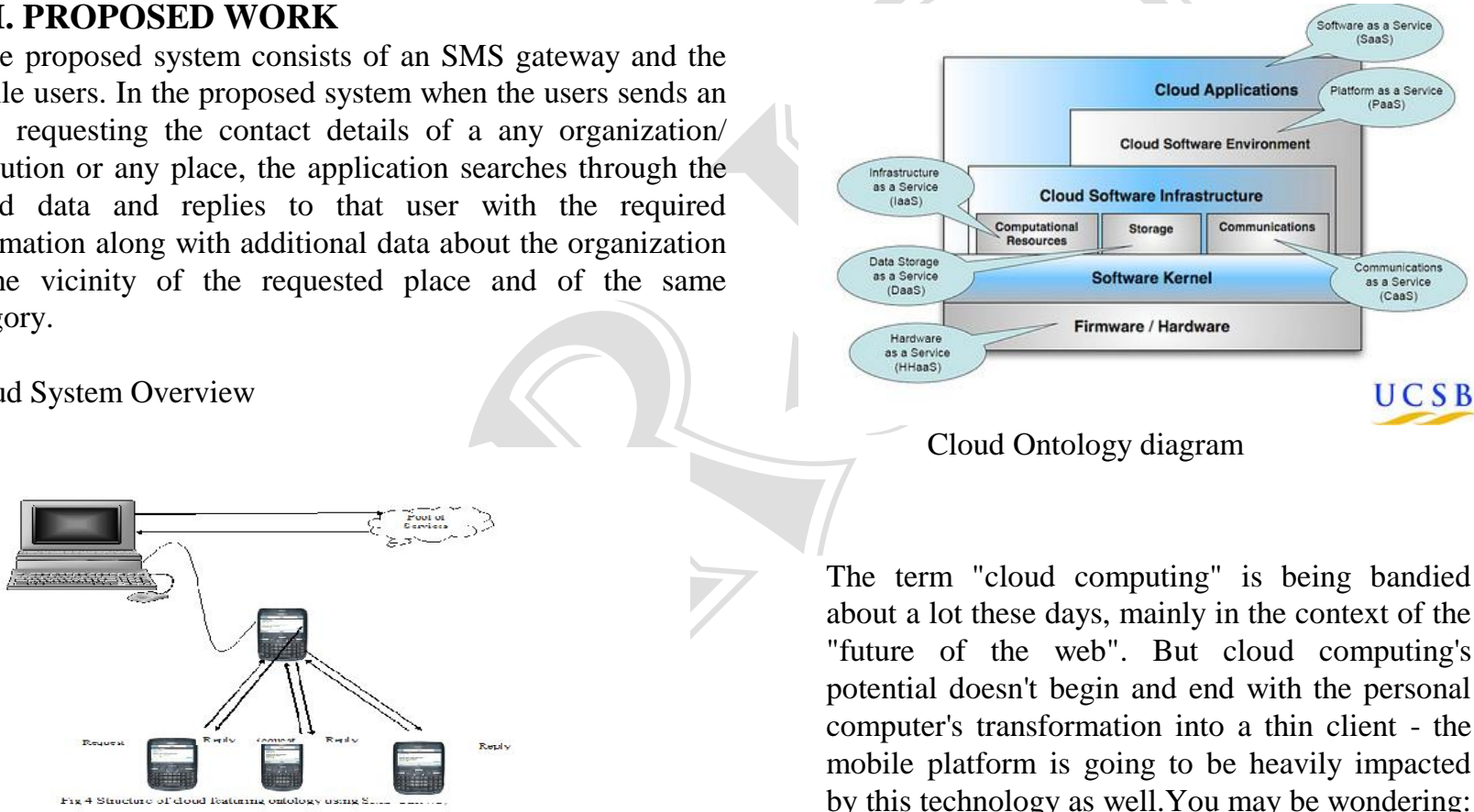

The term "cloud computing" is being bandied about a lot these days, mainly in the context of the "future of the web". But cloud computing's potential doesn't begin and end with the personal computer's transformation into a thin client - the mobile platform is going to be heavily impacted by this technology as well.You may be wondering: what does the term "mobile cloud computing" really mean? Basically, it refers to an infrastructure where both the data storage and the data processing happen outside of the mobile device. Today, there are already some good examples of mobile cloud computing applications including mobile Gmail, Google Maps, and some navigation apps. However, the majority of applications today still do most of the data storage and processing on the mobile devices themselves and not in the cloud. In a few years, that could change. In this diagram, there are two new services which are used other than the normal four services. The Caas(Communication as a service ) is used for the communication happening between two hand held devices or wireless devices and Daas( Data as a storage )service is for the users to 


\section{Arun Arunachalam,Shreeharsha Chandrashekar / IOSR Journal of Engineering (IOSRJEN) \\ www.iosrjen.org \\ ISSN : 2250-3021}

Vol. 2 Issue 2, Feb.2012, pp.280-283

get the data they requested based upon domain keywords and category.

\section{PROPOSED SOLUTION}

We propose a Cloud computing based Ontology that will provide following features

1) Automatic operation :-

No one is needed to manually read the message of the user then find the information which user requires and then send the reply to the message asking for information on the same mobile number.

Example:

When a message will arrive on the cell phone which is connected to the server computer it will automatically read the message find information and send message again to the user without any human.

\section{2) Need not to remember :-}

Application will help you by providing the information, when you don't have any information or just forget the information about any concept through an SMS.

Example:When you want information like contact number or address of some place like theatre.

\section{3) Personalized location based Search}

This service can send SMS to user's current location with information to help users to find food, lodging, and entertainment to fit their tastes and requirements.

\section{4) Useful :-}

This system will be specially useful for the common people who do not use a high end phone and want to retrieve information at the most economic way.

\section{5) Simple in operation :-}

System is very simple as user just need to send an SMS which is simple operation on any mobile device and known to any person of ant age group.

\section{6) Help user in trouble :-}

2.It will help user who are in trouble and needs immediate help to search and find information wherever and whenever in any emergency within few minutes.

In this the user will send a msg to the sms gateway depending on wat type of queries does the the user have. The gateway will then interact with the server which has cloud application eucalyptus installed in it.And the server will interact with the sms gateway and search the answer for the query based on the ontology concept that searches according to keywords and give more details like the FAQ(frequently asked queries ).And thn the server will send the answer to the sms gateway which will thn send it to the desired user who has the query. Cloud computing featuring Ontology provides users of mobile devices personalized services tailored to their current location. Ontology is a formal specification of a shared conceptualisation. Using the Ontology search interface, the user searches a registry of Ontologies based upon domain, keywords, category or another searchable metadata field. In general, cloud computing customers do not own the physical infrastructure, instead avoiding capital expenditure by renting usage from a third-party provider. They consume resources as a service and pay only for resources that they use. In this case user is only charged as per the messaging scheme he is using. User can access this service anywhere anytime. Since this service is deployed on cloud, it is going to offer complete location transparency. There won't be any need to maintain physical servers thus helping in cost reduction. Also the issue about storage is taken care of by the service provider itself.

\section{VI .CONCLUSION}

The dramatic rise in the price of the high end phones has led us to the development of this application. The prices of the smart phones have hiked and are not affordable to all the classes of users.For simply searching for information user has to go through a series of several steps and in a manual process also there are high chances of Human error. The application performs a simple search operation by replying to the users requested information in form of a Short Message Service 
Vol. 2 Issue 2, Feb.2012, pp.280-283

popularly known as SMS.When the user sends a message to the application requesting for some information, the application first process the users message. It then creates a log. The message is processed and broken down according to its semantics. The application then searches for the information and replies back with the correct information.The msg first reaches the sms gateway and then through the gateway it reaches the server and then with the help of the ontology concept the solution to the query is processed by the server and then send to the sms gateway. Which then forwards it to the desired user.

\section{REFERENCES}

1. Rosemann, M., \& Recker, J. (2006). "Context-aware process design: Exploring the extrinsic drivers for process flexibility". In T. Latour \& M. Petit. 18th international conference on advanced information systems engineering. proceedings of workshops and doctoral consortium. Luxembourg: Namur University Press. pp. 149-158.

2. Kaltz, J.W., Ziegler, J., Lohmann, S. (1995). "Context-aware Web Engineering: Modeling and Applications" (PDF). Revue d 'Intelligence Artificial 19 (3): 439-458.

3. B. Schilit, N. Adams, and R. Want. (1994). "Context-aware computing applications" (PDF). IEEE Workshop on Mobile Computing Systems and Applications (WMCSA'94), Santa Cruz, CA, US. pp. 89-101.

http://sandbox.parc.com/want/papers/parctab-wmcdec94.pdf.

4. Schilit, B.N. and Theimer, M.M. (1994). "Disseminating Active Map Information to Mobile Hosts". IEEE Network 8 (5): 22-32. doi:10.1109/65.313011.

5. Context and Adaptively in Pervasive Computing Environments: Links with Software Engineering and Ontological Engineering. A. Soylu, P. De Causmaecker, P. Desmet. Journal of Software, Vol 4, No 9 (2009), 992-1013, Nov 2009 doi:10.4304/jsw.4.9.992-1013.

6. "Ontological Approach toward Cybersecurity in Cloud Computing" Takeshi Takahashi National Institute of Youki KadobayashiNara Institute of Science and Hiroyuki FujiwaraSolution Crew Inc.

7. "An Ontology-Supported Ubiquious Interface Agent for CLOUD COMPUTING” SHENG-YUAN YANG1..CHUN-LIANG HSU2, DONG-LIANG LEE3 1Department of Computer and Communication Engineering, St. John's University, Taiwan2Department of Electrical Engineering, St. John's University, Taiwan

8. "T. R. Gruber. Toward principles for the design of ontologies used for knowledge sharing". International Journal of Human-Computer Studies. 